\title{
A new single nucleotide polymorphism in the ryanodine gene of chicken skeletal muscle
}

\author{
A.A. Droval ${ }^{1}$, E. Binneck ${ }^{2}$, S.R.R. Marin ${ }^{2}$, F.G. Paião ${ }^{1}$, A. Oba $^{1}$, \\ A.L. Nepomuceno ${ }^{2}$ and M. Shimokomaki ${ }^{1,3}$ \\ ${ }^{1}$ Departamento de Ciência e Tecnologia de Alimentos, \\ Universidade Estadual de Londrina, Londrina, PR, Brasil \\ ${ }^{2}$ Laboratório de Biotecnologia Vegetal, \\ Empresa Brasileira de Pesquisa Agropecuária-Soja, Londrina, PR, Brasil \\ ${ }^{3}$ Universidade Tecnológica Federal do Paraná, Campus Londrina, \\ Londrina, PR, Brasil \\ Corresponding author: M. Shimokomaki \\ E-mail: mshimo@uel.br
}

Genet. Mol. Res. 11 (2): 821-829 (2012)

Received May 30, 2011

Accepted December 13, 2011

Published April 3, 2012

DOI http://dx.doi.org/10.4238/2012.April.3.4

\begin{abstract}
Some genes affect meat quality in chickens. We looked for polymorphisms in the Gallus gallus $\alpha$-RyR gene (homologous to RyR 1) that could be associated with PSE (pale, soft and exudative) meat. Because RyR genes are over 100,000 bp long and code for proteins with about 5000 amino acids, primers were designed to amplify a fragment of hotspot region 2, a region with a high density of mutations in other species. Total blood DNA was extracted from 50 birds, 25 that had PSE meat and 25 normal chickens. The DNA samples were amplified by PCR, cloned, sequenced, and used to identify single nucleotide polymorphisms (SNPs). The amplified fragment of $\alpha$-RyR was 604 nucleotides in length; 181 nucleotides were similar to two exons from a hypothetical turkey cDNA sequence for $\alpha$-RyR. A non-synonymous nucleotide substitution (G/A) was identified in at least one of the three sequenced clones obtained from nine animals, six PSE (HAL+) birds and three normal (HAL-) birds; they were heterozygous for this mutation.
\end{abstract}


This SNP causes a change from Val to Met in the $\alpha$-RYR protein. Since the frequencies of this SNP were not significantly different in the PSE versus normal chickens, it appears that this mutation (in heterozygosity) does not alter the structure or function of the muscle protein, making it an inappropriate candidate as a genetic marker for PSE meat.

Key words: Mutation; Meat quality; SNP; Ryanodine receptor protein

\section{INTRODUCTION}

Pale, soft and exudative (PSE) meat is characterized by its pale color, soft texture, and poor water-holding capacity (WHC) (Candek-Potokar et al., 1998; Brewer and McKeith, 1999; Wilhelm et al., 2010). In Brazil, the incidence of PSE meat in the poultry industry is approximately $22 \%$ (Soares et al., 2003), depending on how stressful conditions are for the birds prior to slaughter. The demand for chicken products by consumers has gradually shifted from whole carcasses to processed products that currently represent a larger proportion of the chicken meat market. Refrigerated carcasses containing PSE meat accumulate exudates, in particular during thawing, resulting in a noticeably excessive amount of fluid after packaging. When these abnormal samples are used as the raw material for meat emulsions, they generate meat products with defects (Simões et al., 2009). For example, mortadella prepared with PSE meat has lower WHC, altered texture and lower emulsion stability, and it requires additives to restore the functional properties of normal meat (Kissel et al., 2009).

The causes of PSE meat seem to be both genetic and environmental. In pigs, the genetic cause of this type of meat was identified as a point mutation in the RyR 1 gene at nucleotide 1843, which is responsible for an amino acid substitution (Arg615 to Cys615) in the ryanodine receptor (RYR), a $\mathrm{Ca}^{2+}$-channel protein found in the skeletal muscle sarcoplasmic reticulum (Fujii et al., 1991). This mutation leads to an alteration in $\mathrm{Ca}^{2+}$ homeostasis, hypermetabolism, intense muscle contraction, and malignant hyperthermia in stressed porcine stress syndrome-susceptible pigs (Wang et al., 1999). The physiological consequences of increasing resting $\mathrm{Ca}^{2+}$ levels before slaughter include the promotion of muscle hypermetabolism, leading to post-mortem heat production and acidosis in muscle tissue. The RyR 1 gene is commonly referred to as the halothane gene because pigs with mutant alleles can be detected by exposure to halothane (Louis et al., 1993). The use of this anesthetic compound has proven to be effective for identifying pigs that carry a genetic defect in RyR and are therefore susceptible to developing PSE meat in response to stress. Halothane has been applied to select pigs prone to develop PSE meat, making the mutant version of RyR the first allele to be identified and selected using molecular genetic tools in a pig breeding program (Fujii et al., 1991).

There are three RYR isoforms in mammals, RYR1, RYR2 and RYR3, which are each expressed in different tissues. RYR1 is predominantly expressed in muscle cells, and RYR2 and RYR3 are predominantly expressed in cardiac and brain tissues, respectively (Sutko and Airey, 1996; Chiang et al., 2007, 2008). In birds, skeletal muscle was found to express equal amounts of two isoforms, $\alpha$-RYR and $\beta$-RYR, which are homologous to RYR1 and RYR3, respectively (Ottini et al., 1996). In chickens that develop PSE meat, the expression of the $\alpha$-RyR gene is unchanged, while $\beta$-RyR expression is lower than in chickens with PSE meat (Oda et al., 2009). 
Strasburg and Chiang (2009) mapped out three hotspots in the turkey $\alpha$-RYR protein between amino acids 1-1000, 2000-3000 and 4000-5000. These regions are crucial for the proper functioning of RYRs because they contain highly conserved domains involved in interactions with other regulatory proteins and also constitute the inside portion of the calcium channel. The mutation responsible for PSE in pig and human malignant hyperthermia is located in hotspot 1 (Brini, 2004; Strasburg and Chiang, 2009).

None of the mutations identified in turkey and chicken are the same as the mutation that has been identified in the pig. Therefore, the effects of a defective RYR protein are not yet as well understood in birds as they are in the pig, and how these mutations in RyR lead to PSE meat in poultry is not known (Strasburg and Chiang, 2003, 2009). Ziober et al. $(2009,2010)$ concluded that the expression pattern of $\alpha$-RyR transcripts in broiler chickens is altered by heat stress and halothane treatment, irrespective of PSE occurrence. Although the sequence of the chicken genome is nearly complete (http://www.ensembl.org/Gallus_gallus/Info/Index), the genomic and transcript sequences of chicken $\alpha$-RyR have not yet been fully elucidated. To date, only 2031 nucleotides of the $\alpha$-RyR gene have been sequenced, including the $\mathrm{C}$-terminal region, which contains hotspot 3 , and are available in GenBank (X95266.1) (Ottini et al., 1996). Therefore, the aim of this study was to sequence a fragment located in the hotspot 2 region of the $\alpha$-RyR gene and to identify single nucleotide polymorphisms (SNPs) in broiler chickens that had or had not developed PSE meat.

\section{MATERIAL AND METHODS}

A total of 851 Ross chickens were tested with halothane $48 \mathrm{~h}$ prior to slaughter and were classified as halothane-sensitive (HAL+) or -insensitive (HAL-) in a special experimental anesthetic chamber constructed in our laboratory (Marchi et al., 2009). Each experimental treatment consisted of three birds being placed in the three-hole chamber. The birds were anesthetized with $3.0 \%$ halothane in pure oxygen at a flow rate of $6.0 \mathrm{~L} / \mathrm{min}$ for $5 \mathrm{~min}$. Slaughter was carried out as previously described and consisted of electrical stunning, bleeding, defeathering, evisceration, carcass water cooling, deboning, and chilling (Guarnieri et al., 2004). Aliquots of blood from the jugular vein were placed into tubes containing K3 EDTA (Sigma, St. Louis, MO, USA), stored at $-20^{\circ} \mathrm{C}$ and used for DNA extraction. A total of 154 chicken breast fillets from birds that were previously classified as HAL+ or HAL- were characterized as to whether they contained PSE meat by determining $\mathrm{pH}$, color, and WHC values as described previously (Wilhelm et al., 2010).

Although almost the entire genome of Gallus gallus has been sequenced, only a portion of the C-terminus of the $\alpha$-RyR gene has been deposited and annotated in GenBank. The BLAST program was used (Altshul et al., 1997) to search for chicken sequences similar to a turkey $\alpha$-RyR cDNA (EU177005.1). Four stretches of G. gallus sequence (NW_001476149, NW_001476304, NW_001479482, and NW_001484776) were identified as similar to the turkey $\alpha$-RyR sequence. The NW_001476149 contig was located in the interval between nucleotides 6506 to 7405 in the turkey $\alpha$-RyR transcript, which is within the region described as belonging to hotspot 2 (Strasburg and Chiang, 2009). Thus, the sequence of NW_001476149 was used to design forward (5'-AGGAAGCCGACCTCCAAC-3') and reverse (5'-TGGGGGTCATCAGCCTTC-3') primers using the Primer3Plus program (Untergasser et al., 2007).

Blood from 50 birds was subjected to DNA extraction using the BD DNAzol reagent (Invitrogen, Carlsbad, CA, USA), following manufacturer instructions. A total of 25 DNA samples from normal and PSE samples were used to amplify a 604-bp fragment in polymerase chain reactions 
(PCR) in a volume of $25 \mu \mathrm{L}$. The amplification reactions contained $50 \mathrm{ng}$ chicken DNA, $1 \mathrm{mM}$ forward and reverse primers, $0.1 \mathrm{mM}$ dNTPs, $2.5 \mathrm{mM} \mathrm{MgSO}_{4}, 1 \mathrm{U}$ Platinum ${ }^{\circledR}$ Taq High Fidelity DNA polymerase (Invitrogen) and 1X PCR buffer (60 mM Tris- $\mathrm{SO}_{4}, \mathrm{pH} 8.9$, and $18 \mathrm{mM}$ ammonium sulfate). Amplifications were performed in a DNA thermocycler (VeritiTM/Model 96-Well Thermal Cycler, Applied Biosystems). The reaction mixtures were heated at $95^{\circ} \mathrm{C}$ for $2 \mathrm{~min}$ and subjected to 38 cycles of denaturation at $95^{\circ} \mathrm{C}$ for $30 \mathrm{~s}$, annealing at $65^{\circ} \mathrm{C}$ for $1 \mathrm{~min}$, and extension at $72^{\circ} \mathrm{C}$ for $1.5 \mathrm{~min}$, with an additional $10 \mathrm{~min}$ extension at $72^{\circ} \mathrm{C}$. Products from the multiplex PCR were subjected to electrophoresis on $1.5 \%$ agarose gels, stained with ethidium bromide, examined using a UV transilluminator and photographed. PCR products were cut out of the gel and purified using the Wizard SV Gel kit and PCR Clean-up System (Promega) following manufacturer instructions.

The PCR products eluted from the gel were ligated into a TA cloning vector (Invitrogen). Plasmids were transformed into competent cells of Escherichia coli and grown on LB-agar medium under X-gal and ampicillin selection. Three individual white colonies from each sample that was positive for the presence of inserted DNA (by $\beta$-galactosidase expression) were picked at random and analyzed further. The insert sequence was determined using an ABI PRISM 3100 genetic analyzer (Applied Biosystems) and a Big Dye terminator sequencing kit (Applied Biosystems). The chromatograms were analyzed using the package Vector NTI ContigExpress Suite 8 software (InforMax). The programs blastn and blastx (Altschul et al., 1997) were used to check the sequences obtained for similarity to the RyR sequences from other organisms that are available in GenBank. The samples that contained apparent mutations were submitted to resequencing for confirmation. Finally, the SIFT program (Sorting Intolerant from Tolerant - http:// sift.jcvi.org/) was used to estimate the effects that the mutations might have on protein structure.

\section{RESULTS AND DISCUSSION}

From the 851 experimental birds, 154 breast fillet samples were characterized as containing PSE meat; however, not all of them came from HAL+ birds. In fact, 94 of these breast fillet samples came from HAL + chickens (64.6\%), and 60 breast fillets were from HALchickens, which were not sensitive to halothane.

Of the 50 birds selected for DNA extraction, 25 of them were derived from HAL+, and all of them were characterized as having PSE meat. Twenty-three of 25 HAL- birds did not contain any PSE meat, while the remaining 2 were positive for PSE meat.

Table 1 gives the $\mathrm{pH}, \mathrm{L}^{*}$ (color) and WHC values from the normal and PSE meats from all $50 \mathrm{HAL}+$ and HAL- birds. These values are consistent with previous results (Olivo et al., 2001; Soares et al., 2003; Guarnieri et al., 2004; Wilhelm et al., 2010).

\footnotetext{
Table 1. Average $\mathrm{pH}$, color ( $\left.\mathrm{L}^{*}\right)$ and water-holding capacity (WHC) of fillets from pale, soft and exudate (PSE) and normal birds.

\begin{tabular}{llll}
\hline & $\mathrm{pH}$ & $\mathrm{L}^{*}(0$ to 100$)$ & WHC (\%) \\
\hline PSE $(\mathrm{N}=25)$ & $5.73 \pm 0.03^{\mathrm{b}}$ & $57.19 \pm 0.51^{\mathrm{a}}$ & $68.67 \pm 0.80^{\mathrm{b}}$ \\
Normal $(\mathrm{N}=25)$ & $5.97 \pm 0.03^{\mathrm{a}}$ & $51.39 \pm 0.37^{\mathrm{b}}$ & $73.11 \pm 0.75^{\mathrm{a}}$ \\
\hline
\end{tabular}

Data are reported as means \pm SE. Different superscript letters in same column indicate a significant difference as determined by the Student $t$-test $(\mathrm{P} \leq 0.05)$.

The RyR gene is distinguished by its large size (approximately 100,000 bp) and large number of exons and introns. Less than $10 \%$ of this gene codes for RYR, but this information
} 
is distributed among more than 90 coding exons. This structure makes it difficult to sequence the whole gene because it is usually possible to find only a fraction of the transcript sequence. Sequence information for poultry RyR genes is scarce; for example, for turkey, there is no complete RyR DNA sequence available, only a full-, 15,153-bp sequence of an $\alpha$-RyR cDNA (EU177005.1). Similarly, in chicken, only a 2031-bp portion of the $\alpha$-RyR transcript has been sequenced (Ottini et al., 1996). Therefore, it was only possible to design pairs of primers that could amplify fragments of the chicken $\alpha$-RyR. Only one pair of primers (based on the G. gallus contig NW_001476149) amplified a fragment of the expected size, i.e., about 600 nucleotides of the $\alpha$-RyR gene (Figure 1), adding more known regions for this gene.

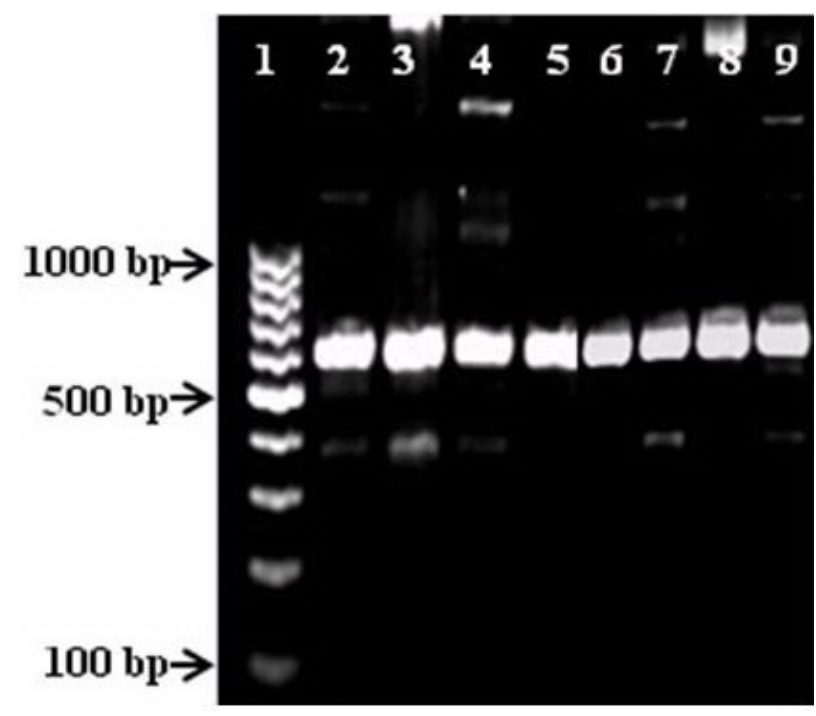

Figure 1. Electrophoresis of amplification products on a $1 \%$ agarose gel. Lane $1=100$-bp molecular weight marker (Invitrogen); lanes 2-5= DNA samples from chickens that have PSE meat; lanes 6-9=DNA samples from chickens with normal meat.

We identified two sequences similar to $\alpha$-RyR that we called alleles I and II, respectively. These two different genomic sequences were deposited in GenBank (accession Nos. HN153277 and HR30866). An SNP was identified at position 585, where it was verified to be a substitution of a $\mathrm{G}$ (guanine) for an A (adenine). Genomic sequence from allelle I was more common in our sample, with $82 \%$ of the birds being homozygous for A at position 585 (Figures 2 and 3). Allele II, which carries the A to G substitution nucleotide at position 585, alters the RYR amino acid sequence relative to the turkey $\alpha$-RyR, changing a valine to a methionine (Figure 3). According to the SIFT program, this substitution may cause changes in protein structure, and this change may be tolerable.

Aligning the chicken $\alpha$-RyR genomic sequence of allele I with the turkey cDNA sequence (EU177005.1) using the bl2seq program (Altschul et al., 1997), we identified at least two partial exons, corresponding to nucleotides 1 to 45 and 467 to 604 , respectively, as well as a complete intron (nucleotides 46-466). The sequence comprising the spliced exons (183 nucleotides) was designated the chicken coding region (CR) (Figure 4). 
571

585

604

Chicken 09 PSE Clone 1 AGTTCCTCCTCCACGTGTTGGAGGTCGGCTTCCT Clone 2 AGTTCCTCCTCCACGTGTTGGAGGTCGGCTTCCT

Clone 3 AGTTCCTCCTCCACATGTTGGAGGTCGGCTTCCT

Chicken 10 PSE clone 1 AGTTCCTCCTCCACGTGTTGGAGGTCGGCTTCCT

Clone 2 AGTTCCTCCTCCACGTGTTGGAGGTCGGCTTCCT

Clone 3 AGTTCCTCCTCCACATGTTGGAGGTCGGCTTCCT

Chicken 17_PSE clone 1 AGTTCCTCCTCCACATGITGGAGGTCGGTTCCT

Clone 2 AGTTCCTCCTCCACATGTTGGAGGTCGGCTTCCT

Clone 3 AGTTCCTCCTCCACATGTTGGAGGTCGGCTTCCT

Chicken 18_pSE Clone 1 AGTTCCTCCTCCACATGTTGGAGGTCGGCTTCCT

clone 2 AGTTCCTCCTCCACGTGTTGGAGGTCGGCTTCCT

Clone 3 AGTTCCTCCTCCACATGTTGGAGGTCGGCTTCCT

Chicken 19_PSE clone 1 AGTTCCTCCTCCACGTGTTGGAGGTCGGCTTCCT

Clone 2 AGTTCCTCCTCCACGTGTTGGAGGTCGGCTTCCT

Clone 3 AGTTCCTCCTCCACATGTTGGAGGTCGGCTTCCT

Chicken 20_PSE Clone 1 AGTTCCTCCTCCACATGTTGGAGGTCGGCTTCCT

Clone 2 AGTTCCTCCTCCACATGTTGGAGGTCGGCTTCCT

Clone 3 AGTTCCTCCTCCACGTGTTGGAGGTCGGCTTCCT

Chicken 40 Nor Clone 1 AGTTCCTCCTCCACATGTTGGAGGTCGGCTTCCT

Clone 2 AGTTCCTCCTCCACGTGTTGGAGGTCGGCTTCCT

clone 3 AGTTCCTCCTCCACGTGTTGGAGGTCGGCTTCCT

Chicken 45 Nor Clone 1 AGTTCCTCCTCCACATGTTGGAGGTCGGCTTCCT

Clone 2 AGTTCCTCCTCCACGTGTTGGAGGTCGGCTTCCT

Clone 3 AGTTCCTCCTCCACATGTTGGAGGTCGGCTTCCT

Chicken 50_Nor Clone 1 AGTTCCTCCTCCACGTGTTGGAGGTCGGCTTCCT

Clone 2 AGTTCCTCCTCCACGTGITGGAGGTCGGCTTCCT

clone 3 AGTTCCTCCTCCACATGTTGGAGGTCGGCTTCCT

Figure 2. Alignment of the partial nucleotide sequences from nine chickens that carried a polymorphism, both genomic sequences (alleles I and II) at position 585 of the $\alpha$-RyR gene fragment. The polymorphic nucleotide position within each sequence is shown in bold.

By comparison, there were 13 different nucleotides in sequence for $\alpha$-RyR chicken allele I in relation to turkey (EU177705) and 14 different nucleotides in the sequence for $\alpha$-RyR chicken allele II. Although we found differences between chicken allele I and turkey DNA, the alignment of the predicted protein from this sequence region showed $100 \%$ identity with the sequence of the turkey $\alpha$-RYR protein (ABY50125). We also observed high sequence similarity between the chicken $\alpha$-RYR and homologous proteins of frog, rat, pig, rabbit, and human. The degree of identity between species was $84 \%$ with frog and $81 \%$ with the mammalian species (Figure 5 ). The polymorphic sequence showed $96 \%$ identity with the sequence of the turkey $\alpha$-RYR protein and lower similarity with other organisms, i.e., $83 \%$ with frog and $78 \%$ with mammalian species. 


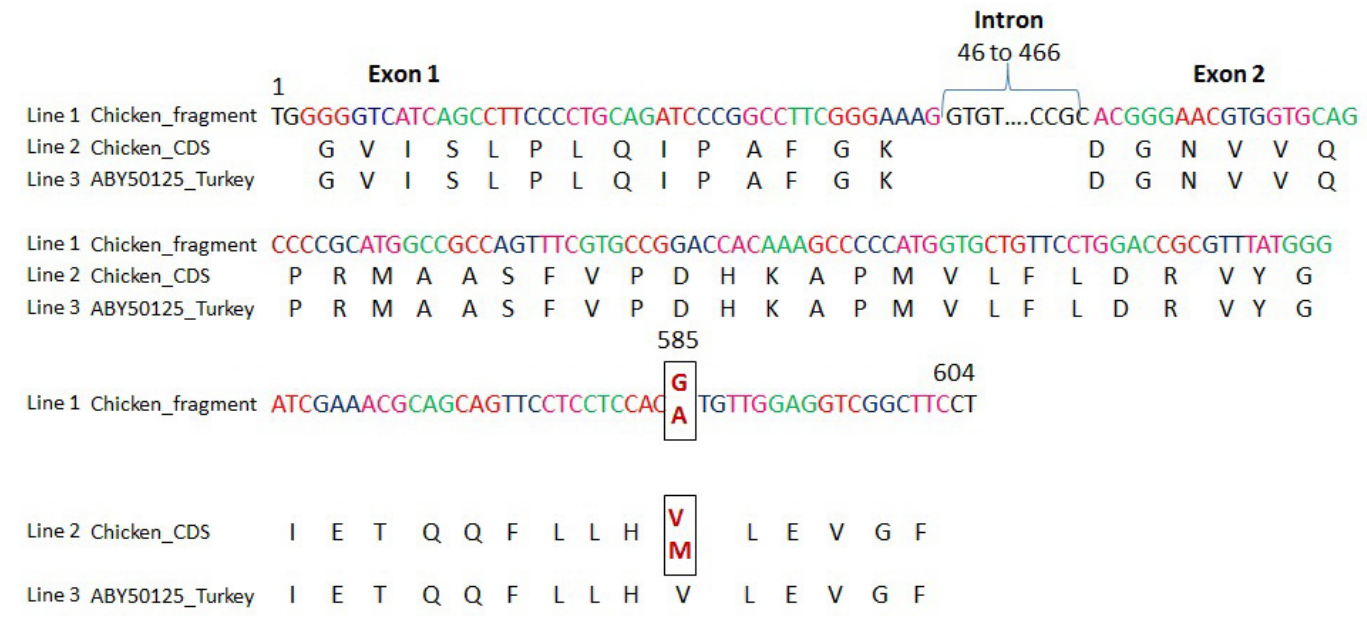

Figure 3. Alignment of fragment nucleotide sequences with translated amino acid sequences, with the polymorphic nucleotide (position 585) and the changed amino acid within the coding region boxed. Line $1=$ Nucleotides colored red, blue, pink, and green correspond to the codons in the coding region, while those colored black correspond to the non-coding region. Line $2=$ The amino acid sequence of a portion (amino acids 2455-2513) of the chicken $\alpha$-RyR protein, with the altered amino acid position shown in red. Line $3=$ The amino acid sequence of the corresponding portion of the turkey $\alpha-R Y R$ protein.

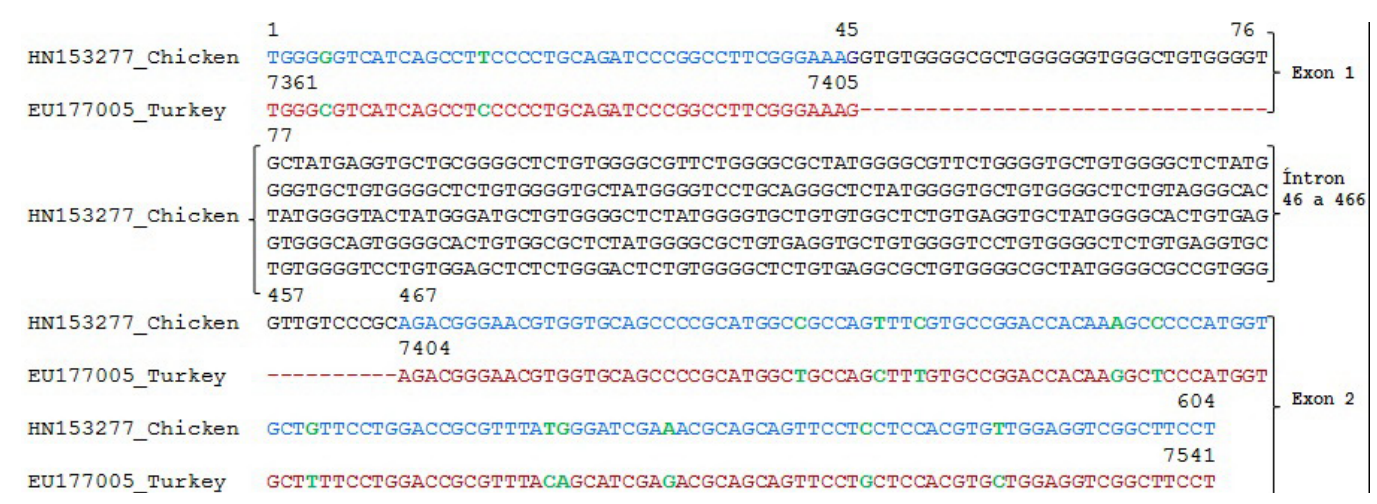

Figure 4. Alignment of the sequenced 604-bp fragment of chicken allele I (HN153277) with the sequences of exons 1 and 2 from the turkey $\alpha$-RyR cDNAs. The position of coding region within the chicken sequence fragment is shown in blue letters. Exons 1 (1-45) and 2 (467-604) from the partial sequence of the turkey $\alpha$ RyR cDNA are shown in red letters, with green letters indicating positions that differ between the chicken and turkey sequences.

When compared to other species, the sequence amplified by us is located at the end of hotspot 2 in the turkey $\alpha$-RyR gene, which is located in a conserved region. Region 1 (1-1000 amino acids), region 2 (2000-3000) and region 3 (4000-5000) are conserved between species (Brini, 2004; Chiang et al., 2007; Strasburg and Chiang, 2009). Many diseases in humans have been linked to mutations located in regions 1 and 2 of the RyR1 gene (Brini, 2004), and according to Chiang et al. (2004), more than 20 mutations that cause amino acid changes or that delete amino acids are located in these regions. 
Brewer MS and McKeith FK (1999). Consumer-rated quality characteristics as related to purchase intent of fresh pork. $J$. Food Sci. 64: 171-174.

Brini M (2004). Ryanodine receptor defects in muscle genetic diseases. Biochem. Biophys. Res. Commun. 322: 1245-1255.

Candek-Potokar M, Zlender B, Lefaucheur L and Bonneau M (1998). Effects of age and/or weight at slaughter on longissimus dorsi muscle: biochemical traits and sensory quality in pigs. Meat Sci. 48: 287-300.

Chiang W, Allison CP, Linz JE and Strasburg GM (2004). Identification of two alpha RyR alleles and characterization of alpha RyR transcript variants in turkey skeletal muscle. Gene 330: 177-184.

Chiang W, Yoon HJ, Linz JE, Airey JA, et al. (2007). Divergent mechanisms in generating molecular variations of alpha RYR and beta RYR in turkey skeletal muscle. J. Muscle Res. Cell Motil. 28: 343-354.

Chiang W, Booren A and Strasburg G (2008). The effect of heat stress on thyroid hormone response and meat quality in turkeys of two genetic lines. Meat Sci. 80: 615-622.

Fujii J, Otsu K, Zorzato F, de Leon S, et al. (1991). Identification of a mutation in porcine ryanodine receptor associated with malignant hyperthermia. Science 253: 448-451.

Guarnieri PD, Soares AL, Olivo R, Schneider JP, et al. (2004). Preslaughter handling with water shower spray inhibits PSE (pale, soft, exudative) broiler breast meat in a commercial plant. Biochemical and ultrastructural observations. J. Food Biochem. 28: 269-277.

Kissel C, Soares AL, Rossa A and Shimokomaki M (2009). Functional properties of PSE (pale, soft, exudative) broiler meat in the production of mortadella. Braz. Arch. Biol. Tech. 52: 213-217.

Louis CF, Rempel WE and Mickelson JR (1993). Porcine Stress Syndrome: Biochemical and Genetic Basis of this Inherited Syndrome of Skeletal Muscle. In: Proceedings of the Annual Reciprocal Meat Conference. National Live Stock and Meat Board, Chicago, 89-96.

Marchi DF, Trindade MA, Oba A, Soares AL, et al. (2009). Sensitivity to halothane and its relationship to the development of PSE (pale, soft, exudative) meat in female lineage broilers. Braz. Arch. Biol. Technol. 52: 219-223.

Oda SHI, Nepomuceno AL, Ledur MC, Oliveira MCN, et al. (2009). Quantitative differential expression of alpha and beta ryanodine receptor genes in PSE (pale, soft, exudative) meat from two chicken lines: broiler and layer. Braz. Arch. Biol. Technol. 52: 1519-1525.

Olivo R, Scares AL, Ida EI and Shimokomaki M (2001). Dietary vitamin E inhibits poultry PSE and improves meat functional properties. J. Food Biochem. 25: 271-283.

Ottini L, Marziali G, Conti A, Charlesworth A, et al. (1996). Alpha and beta isoforms of ryanodine receptor from chicken skeletal muscle are the homologues of mammalian RyR1 and RyR3. Biochem. J. 315 (Pt 1): 207-216.

Sanger Institute (2010). Available at [http://pfam.sanger.ac.uk/family?PF01365]. Accessed October 10, 2010.

Simões GS, Oba A, Matsuo T, Rossa A, et al. (2009). Vehicle thermal microclimate evaluation during Brazilian summer broiler transport and the occurrence of PSE (pale, soft, exudative) meat. Braz. Arch. Biol. Technol. 52: 195-204.

Soares AL, Ida EI, Miyamoto S, Hernández-Blazquez J, et al. (2003). Phospholipase A2 activity in poultry PSE, pale, soft, exudative, meat. J. Food Biochem. 27: 309-320.

Strasburg GM and Chiang W (2003). Genetic Basis for Pale, Soft and Exudative Turkey Meat. In: Proceedings of the 56th American Meat Science Association. American Meat Science Association, Columbia, 17-22.

Strasburg GM and Chiang W (2009). Pale, soft, exudative turkey - The role of ryanodine receptor variation in meat quality. Poult. Sci. 88: 1497-1505.

Sutko JL and Airey JA (1996). Ryanodine receptor $\mathrm{Ca}^{2+}$ release channels: does diversity in form equal diversity in function. Phys. Rev. 76: 1027-1071.

Untergasser A, Nijveen H, Xiangyu R, Bisseling T, et al. (2007). Primer3Plus, an enhanced web interface to Primer3. Nucleic Acids Res. 35: W71-W74.

Wang LJ, Byrem TM, Zarosley J, Booren AM, et al. (1999). Skeletal muscle calcium channel ryanodine binding activity in genetically unimproved and commercial turkey populations. Poult. Sci. 78: 792-797.

Wilhelm AE, Magaghini MB, Hernández-Blazquez FJ, Ida EI, et al. (2010). Protease activity and the ultrastructure of broiler chicken PSE (pale, soft, exudative) meat. Food Chem. 119: 1201-1204.

Ziober IL, Paião FG, Marin SRR, Marchi DF, et al. (2009). Molecular cloning of $\alpha$ RYR hotspot region 1 from broiler chicken. Braz. Arch. Biol. Technol. 52: 225-231.

Ziober IL, Paiao FG, Marchi DF, Coutinho LL, et al. (2010). Heat and chemical stress modulate the expression of the alpha-RYR gene in broiler chickens. Genet. Mol. Res. 9: 1258-1266. 